BMJ Open

Diabetes

Research

\& Care

\title{
Poorly controlled diabetes mellitus alters placental structure, efficiency, and plasticity
}

\author{
Jackson Nteeba, ${ }^{1,2}$ Kaela M Varberg (D) ,1,2 Regan L Scott, ${ }^{1,2}$ Mikaela E Simon, ${ }^{1,2}$ \\ Khursheed lqbal, ${ }^{1,2}$ Michael J Soares ${ }^{1,2,3,4,5}$
}

\begin{abstract}
To cite: Nteeba J, Varberg KM, Scott RL, et al. Poorly controlled diabetes mellitus alters placental structure, efficiency, and plasticity. BMJ Open Diab Res Care 2020;8:e001243. doi:10.1136/ bmjdrc-2020-001243
\end{abstract}

- Additional material is published online only. To view please visit the journal online (http://dx.doi.org/10.1136/ bmjdrc-2020-001243).

JN and KMV contributed equally.

Received 3 February 2020

Revised 11 May 2020

Accepted 21 May 2020
Check for updates

(C) Author(s) (or their employer(s)) 2020. Re-use permitted under CC BY-NC. No commercial re-use. See rights and permissions. Published by BMJ.

For numbered affiliations see end of article.

Correspondence to Dr Kaela M Varberg; kvarberg@kumc.edu and Dr Michael J Soares; msoares@kumc.edu

\section{ABSTRACT}

Introduction The hemochorial placenta provides a critical barrier at the maternal-fetal interface to modulate maternal immune tolerance and enable gas and nutrient exchange between mother and conceptus. Pregnancy outcomes are adversely affected by diabetes mellitus; however, the effects of poorly controlled diabetes on placental formation, and subsequently fetal development, are not fully understood.

Research design and methods Streptozotocin was used to induce hyperglycemia in pregnant rats for the purpose of investigating the impact of poorly controlled diabetes on placental formation and fetal development. The experimental paradigm of hypoxia exposure in the pregnant rat was also used to assess properties of placental plasticity. Euglycemic and hyperglycemic rats were exposed to ambient conditions ( $21 \%$ oxygen) or hypoxia (10.5\% oxygen) beginning on gestation day (gd) 6.5 and sacrificed on gd 13.5. To determine whether the interaction of hyperglycemia and hypoxia was directly altering trophoblast lineage development, rat trophoblast stem (TS) cells were cultured in high glucose $(25 \mathrm{mM})$ and/ or exposed to low oxygen $(0.5 \%$ to $1.5 \%)$.

Results Diabetes caused placentomegaly and placental malformation, decreasing placental efficiency and fetal size. Elevated glucose disrupted rat TS cell differentiation in vitro. Evidence of altered trophoblast differentiation was also observed in vivo, as hyperglycemia affected the junctional zone transcriptome and interfered with intrauterine trophoblast invasion and uterine spiral artery remodeling. When exposed to hypoxia, hyperglycemic rats showed decreased proliferation and ectoplacental cone development on gd 9.5 and complete pregnancy loss by gd 13.5. Furthermore, elevated glucose concentrations inhibited TS cell responses to hypoxia in vitro.

Conclusions Overall, these results indicate that alterations in placental development, efficiency, and plasticity could contribute to the suboptimal fetal outcomes in offspring from pregnancies complicated by poorly controlled diabetes.

\section{INTRODUCTION}

The impact of diabetes on pregnancy outcomes has become an increasing health concern. ${ }^{1}$ During pregnancy maternal glucose can cross the placenta, which results in fetal exposure to hyperglycemia during critical stages of intrauterine development. ${ }^{2}$

\section{Significance of this study}

What is already known about this subject?

Pregnancy outcomes are adversely affected by diabetes mellitus.

- Successful pregnancy in the human requires deep trophoblast cell invasion and extensive spiral artery remodeling to facilitate optimal maternal blood delivery to the developing conceptus.

What are the new findings?

- Diabetes-induced placental malformation decreases placental efficiency and fetal size.

- Hyperglycemia disrupts rat trophoblast stem cell differentiation into invasive trophoblast.

- Diabetes impairs intrauterine trophoblast invasion.

- Hyperglycemia adversely affects placental adaptations to hypoxia.

- High glucose blunts trophoblast stem cell responses to low oxygen.

How might these results change the focus of research or clinical practice?

> Investigating placental development in the context of disease is imperative to the discovery of future preventative and therapeutic strategies.

Increasing evidence supports the idea that pregnancy complications and poor fetal outcomes associated with diabetes could be linked to defects in placental development and function. ${ }^{3}$ Successful pregnancy in the human requires deep trophoblast cell invasion and extensive spiral artery remodeling to facilitate optimal maternal blood delivery to the developing conceptus. ${ }^{45}$ Numerous physiological and environmental factors can affect trophoblast cell development and, thus, the extent of trophoblast cell-mediated spiral artery remodeling. ${ }^{6}$ Insufficient remodeling can then lead to the development of severe pregnancy complications. Thus, investigating placental development in the context of disease is imperative to the discovery of future preventative and therapeutic strategies. 
In the current study, our goals were to investigate regulatory events mediating the deleterious effects of diabetes on placental development and to evaluate the impact of hyperglycemia on placental plasticity. To facilitate our investigation, we administered streptozotocin (STZ) to pregnant rats on gestation day (gd) 6.5 to induce diabetes during pregnancy. ${ }^{8}$ STZ was first described as a model of diabetes mellitus in 1976, due to its ability to induce nearly complete beta cell destruction. ${ }^{9} \mathrm{STZ}$ is primarily considered a model of type 1 diabetes mellitus because it targets and inactivates beta cells. ${ }^{10}$ STZ has also been used in the context of pregnancy to study the consequences of maternal glucose intolerance on fetal outcomes. ${ }^{11}{ }^{12}$ Using the STZ model, we show that intrauterine exposure to hyperglycemia alters placental morphology, efficiency, and plasticity. Thus, inadequate placental function in mothers with poorly controlled diabetes could contribute to poor fetal outcomes.

\section{RESEARCH DESIGN AND METHODS}

\section{Animal care and treatment specifications}

Holtzman Sprague-Dawley rats were housed in an environmentally controlled facility at the University of Kansas Medical Center (KUMC) with lights on from 0600 to 2000 hours and allowed free access to food and water. Female rats (8-10 weeks of age) were mated with adult male rats ( $>3$ months of age). Mating was assessed by inspection of vaginal lavages. The presence of sperm in the vagina was designated gd 0.5. A single dose of either STZ ( $45 \mathrm{mg} / \mathrm{kg}$ body weight) or vehicle control solution (0.1 M citrate buffer, $\mathrm{pH} 4.2$ ) was administered via tail vein injection on $\mathrm{gd} 6.5$ so as not to negatively impact embryo implantation. In some experiments, STZ and vehicle control-treated pregnant rats were placed in a hypoxic ( $10.5 \%$ ( $\mathrm{vol} / \mathrm{vol}$ ) oxygen) gas-regulated chamber (BioSpherix, Lacona, New York, USA) from gd 6.5 to 9.5 or gd 6.5 to 13.5 , which represent critical windows in placental development. Pregnant rats housed in ambient conditions ( $21 \%$ (vol/vol) oxygen) served as controls.

\section{Blood glucose measurements}

Body weights and blood glucose were determined prior to and after treatment to ensure equal dosage and hyperglycemia, respectively. Blood glucose levels were measured from the tail vein using the OneTouch Ultra Smart blood glucose monitoring system (LifeScan, Milpitas, California, USA). ${ }^{13}$

\section{Tissue collection}

Rat pancreas, spleen, liver, and placental tissues were collected and weighed on gd 13.5 and gd 18.5. Entire placentation sites, including the ectoplacental cone, were dissected on gd 9.5. Placentation sites were dissected into junctional zone, labyrinth zone, and metrial gland on gd 13.5 and 18.5 , as previously described. ${ }^{14}$ Selection of gds for analysis represented three critical stages of placentation: (1) the onset of placentation, gd 9.5, (2) establishment of placental zonation, gd 13.5, and (3) robust infiltration of invasive trophoblast cells into the uterine parenchyma, gd 18.5. Placentation sites were frozen in dry-ice cooled heptane for histological and morphometric analyses or dissected placentation site compartments snap-frozen in liquid nitrogen and stored at $-80^{\circ} \mathrm{C}$ until processing for biochemical analyses.

\section{Rat trophoblast stem (TS) cell culture}

Blastocyst-derived rat TS cells ${ }^{15}$ were cultured in basal culture medium (RPMI 1640 (Cellgro), 20\% fetal bovine serum (Sigma-Aldrich), $100 \mu \mathrm{M} \quad$ 2-mercaptoethanol (M7522; Sigma-Aldrich), $1 \mathrm{mM}$ sodium pyruvate (ThermoFisher, 11360-070), $50 \mu \mathrm{M}$ penicillin (15140122; ThermoFisher), and $50 \mathrm{U} / \mathrm{mL}$ streptomycin (15140122; ThermoFisher)) supplemented with $70 \%$ rat embryonic fibroblast-conditioned medium, fibroblast growth factor 4 (25 ng/mL; Sigma-Aldrich), and heparin $(1 \mu \mathrm{g} / \mathrm{mL}$; Sigma-Aldrich). To model hyperglycemia, $25 \mathrm{mM}$ glucose (Sigma-Aldrich) was added to the culture medium. D-mannitol (Sigma-Aldrich) was added to the culture medium to control for osmolality. Rat TS cells were exposed to ambient or low oxygen $\left(0.5 \%\right.$ or $\left.1.5 \% \mathrm{O}_{2}\right)$ tensions and $5 \% \mathrm{CO}_{2}$ at $37^{\circ} \mathrm{C}$ in an NAPCO 8000 incubator (ThermoFisher) for 24 hours and then harvested for immunocytochemical or biochemical analyses.

\section{Immunohistochemistry and immunocytochemistry}

Frozen placental tissues were sectioned at a thickness of $10 \mu \mathrm{m}$ with a cryostat. Immunohistochemical analysis was performed on placental sections by immunofluorescence detection using a pan-cytokeratin primary antibody (1:250, F3418; Sigma-Aldrich) and Alexa 488-conjugated goat-antimouse secondary antibody (1:200, A11001; ThermoFisher). Nuclei were visualized with 4',6-diamidino-2-phenylindole (Molecular Probes, Eugene, Oregon, USA). Rat TS cells were fixed with $4 \%$ paraformaldehyde (Sigma-Aldrich) for $20 \mathrm{~min}$ at room temperature. Immunocytochemical analysis was performed by immunofluorescence detection using a primary antibody against prolactin family 8 , subfamily A, member 5 (PRL8A5, 1:200 ${ }^{16}$ ) followed by Alexa 568-conjugated goat-anti-rabbit secondary antibody (1:200, A11001; ThermoFisher). Images were captured on a Nikon 80i upright microscope (Nikon, Melville, New York, USA) with a Photometrics CoolSNAP-ES monochrome camera (Roper, Sarasota, Florida, USA).

\section{Morphometric measurements}

Depth and extent of invasion and size of placental compartments were performed using ImageJ (National Institutes of Health (NIH)) as previously described. ${ }^{17} 18$ Briefly, the depth of intrauterine trophoblast invasion was quantified as an invasion index, defined as the distance of distal-most cytokeratin-positive cell relative to the trophoblast giant cell layer of the placenta divided by the total distance from the giant cell layer to the outer mesometrial uterine surface. The extent of intrauterine trophoblast invasion was determined as the surface area 
within the uterine mesometrial compartment containing cytokeratin-positive trophoblast cells. Junctional zone thickness was estimated as the area intensely staining with antibodies to pan-cytokeratin in placental cross sections. All morphometric measurements were acquired from a histological plane at the center of each placentation site perpendicular to the fetal interface of the placenta.

\section{RNA isolation, complementary DNA (cDNA) synthesis, and transcript measurements}

Total RNA was isolated from tissues using TRIzol reagent (15596018; ThermoFisher). cDNA was synthesized from $1 \mu \mathrm{g}$ of total RNA using the High Capacity cDNA Reverse Transcription Kit (4368813; Applied Biosystems, Foster City, California, USA). Quantitative PCR (qPCR) was performed with a reaction mixture $(20 \mu \mathrm{L})$ containing cDNA diluted five times with water and PowerSYBR Green PCR Master Mix (4367659; Applied Biosystems) using specific primer $(250 \mathrm{nM})$ sequences (online supplementary table 1). Amplification and florescence detection were carried out using an ABI 7500 Real Time PCR system (Applied Biosystems) for 40 cycles $\left(95^{\circ} \mathrm{C}, 10 \mathrm{~min}\right.$; $\left.92^{\circ} \mathrm{C}, 15 \mathrm{~s} ; 60^{\circ} \mathrm{C}, 1 \mathrm{~min} ; 95^{\circ} \mathrm{C}, 15 \mathrm{~s} ; 60^{\circ} \mathrm{C}, 15 \mathrm{~s} ; 95^{\circ} \mathrm{C}, 15 \mathrm{~s}\right)$. Relative transcript expression was calculated by $\Delta \Delta \mathrm{Ct}$ method and normalized to $18 S$ rRNA.

\section{RNA sequencing (RNA-seq)}

Transcript profiles for gd 13.5 rat junctional zone tissue isolated from control and hyperglycemic pregnancies were generated by RNA-seq as previously described. ${ }^{19}$ cDNA libraries from total RNA samples were prepared with Illumina TruSeq RNA preparation kits (Illumina, San Diego, California, USA). Barcoded cDNA libraries were multiplexed and sequenced with a HiSeq2000 DNA sequencer (100 bp paired-end reads) using a TruSeq 200-cycle SBS kit (Illumina) at the KUMC Genome Sequencing Facility. Reads from *.fastq files were mapped to the rat reference genome (Ensembl Rnor_5.0.78) using CLC Genomics Workbench 12.0 (Qiagen, Redwood City, California, USA). mRNA abundance was expressed in reads per kilobase of exon per million reads mapped. A false discovery rate of 0.05 was used as a cut-off for significant differential expression (euglycemia vs hyperglycemia). Functional patterns of transcript expression were analyzed using Ingenuity Pathway Analysis (Qiagen).

\section{Western blotting}

Placental tissues were homogenized in radioimmunopreciptation assay lysis buffer (sc-24948A; Santa Cruz Biotechnology, Dallas, Texas, USA) supplemented with Halt Protease and phosphatase inhibitor cocktail (78443; ThermoFisher). Protein concentrations were determined by the DC protein assay (BioRad, Hercules, California, USA). A total of $50 \mu \mathrm{g}$ of protein per reaction sample were separated on 4\%-20\% ExpressPlus PAGE Gels (M42012, M42015; GenScript, Piscataway, New Jersey, USA), transferred to polyvinylidene fluoride blotting membrane (10600023; GE Healthcare). Following transfer, membranes were blocked in 5\% non-fat milk in Tris-buffered saline with $0.1 \%$ Tween 20, for non-specific binding and subsequently probed with specific primary antibodies to prolactin family 3, subfamily d, member 4 (PRL3D4, 1:500 $\left.{ }^{20}\right)$, PRL8A5 $\left(1: 500^{16}\right)$, actin, beta (ACTB, 1:4000, A1978; Sigma-Aldrich), and glyeraldehyde-3-phosphate dehydrogenase (1:3000, ab9485; Abcam, Cambridge, Massachusetts, USA). Immunoreactive proteins were visualized by Luminata Crescendo Western HRP substrate (WBLUR0500; Millipore, Billerica, Massachusetts, USA) according to the manufacturer's protocol.

\section{Statistical analysis}

Statistical analyses were performed using GraphPad Prism 8 software. Welch's t-tests, Brown-Forsythe and Welch analysis of variance (ANOVA) tests, and two-way ANOVA tests were applied when appropriate. Data are represented as mean $\pm \mathrm{SD}$ with the statistical significance level set at $\mathrm{p}<0.05$.

\section{Data and resource availlability}

The datasets generated and analyzed during the current study are available in the Gene Expression Omnibus website (https://www.ncbi.nlm.nih.gov/geo/; accession no GSE144276). All data generated and analyzed during this study are included in the published article and the online supplementary files. Resources generated and analyzed during the current study are available from the corresponding authors on reasonable request.

\section{RESULTS}

\section{Diabetes-induced placental malformation decreases} placental efficiency and fetal size

To determine the effect of diabetes on hemochorial placentation, pregnant rats were treated with STZ beginning on gd 6.5 to induce hyperglycemia post-implantation but prior to placental formation (figure 1A). This STZ model caused a decrease in maternal body weight on gd $13.5(\mathrm{p}<0.05)$, which is indicative of uncontrolled diabetes (online supplementary figure 1). There were no significant effects of STZ-induced hyperglycemia on maternal pancreas, liver, or spleen weights at gd 13.5 or gd 18.5 (online supplementary figure 1). STZ-treated rats had significantly elevated blood glucose levels compared with vehicle control-treated rats on both gd 13.5 and gd 18.5, indicating that STZ was effectively disrupting beta cell function $(p<0.0001$; figure 1B). Conceptus viability was not significantly different between vehicle control and STZ-treated rats on gd 18.5, despite significantly increased blood glucose levels (figure 1C). To assess the impact of hyperglycemia on placentation and fetal growth, placental and fetal weights were examined on gd 18.5, a developmental state characterized by a fully mature placentation site. Hyperglycemia was associated with significantly 
A

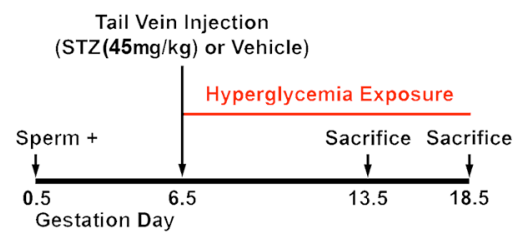

B

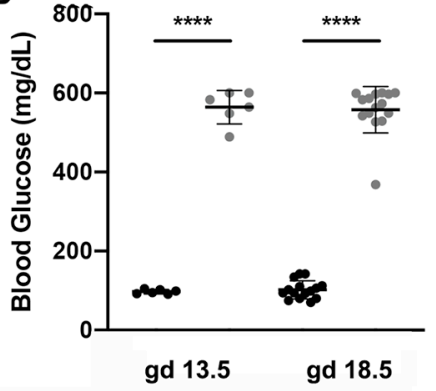

C

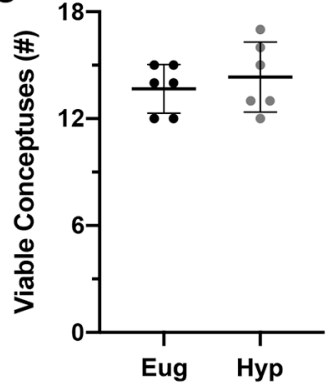

D

Placental Weight

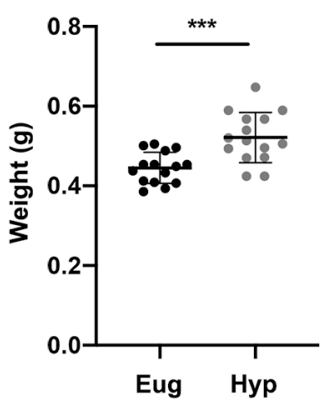

E

Euglycemia

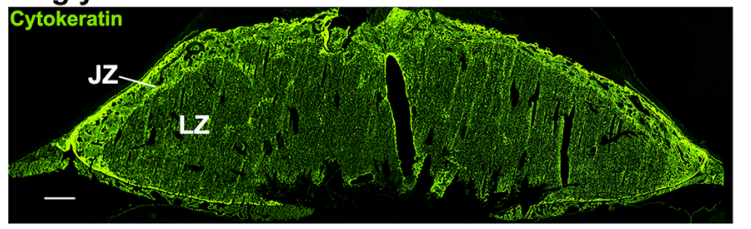

Hyperglycemia

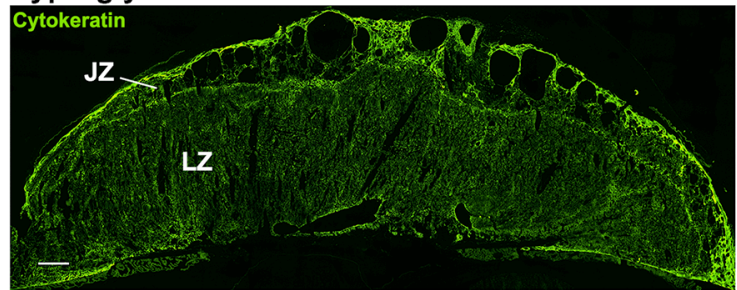

Fetal Weight

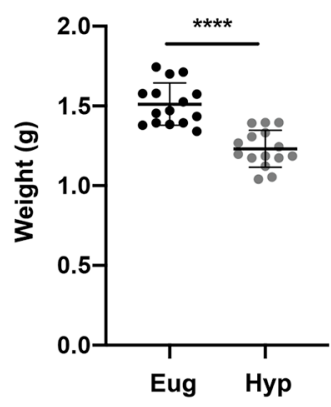

Fetal:Placental Ratio

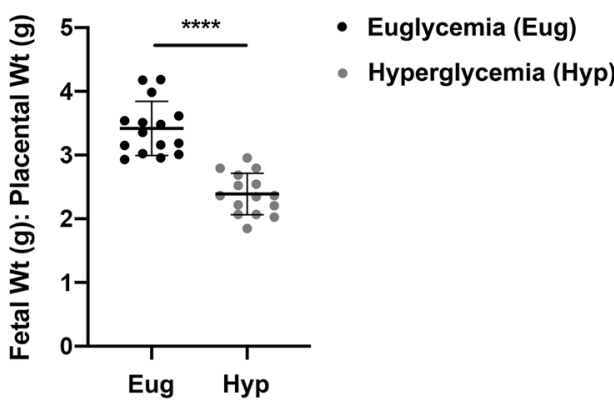

$\mathbf{F}$

Figure 1 Diabetes-induced placental malformation decreases placental efficiency. (A) Schematic depicting the experimental design for streptozotocin (STZ) treatment of pregnant rats. Vehicle control solution (0.1 M citrate buffer, $\mathrm{pH} 4.2)$ or STZ $(45 \mathrm{mg} / \mathrm{kg})$ was injected into pregnant rats on gestation day (gd) 6.5. Rats were subsequently sacrificed for tissue harvest on gd 13.5 or 18.5. (B) Blood glucose was measured on gd 13.5 and 18.5. STZ treatment (gray) significantly increased blood glucose levels compared with vehicle control-treated (black) animals ( $n=6$ per group, $p<0.0001)$. (C) The average number of viable conceptuses was no different between euglycemic and hyperglycemic animals on gd 18.5 ( $n=6$ per group, $p>0.05)$. (D) Placental and fetal weights (g) were measured at the time of sacrifice on gd 18.5. Hyperglycemia significantly increased placental weight $(n=15$ per group; $p<0.001)$, but significantly decreased fetal weight compared with euglycemia ( $n=15$ per group; $p<0.0001)$. The ratio of fetal to placental weight $(g)$, a measure of placental efficiency, was significantly decreased in animals with hyperglycemia $(n=15$ per group; $p<0.0001)$. (E) Placentation sites collected from euglycemic and hyperglycemic pregnant dams on gd 18.5 were sectioned and stained with a cytokeratin-specific antibody (green) for immunohistochemical analysis and visualization of trophoblast cells and placental compartments. (F) Junctional zone (JZ) and labyrinth zone (LZ) areas (pixels) were quantified using ImageJ (National Institutes of Health). Placentae collected from dams with hyperglycemia had significantly increased JZs $(n=10$ Eug, $n=14$ Hyp; $p<0.001)$ and LZs $(n=10$ Eug, $n=14$ Hyp; $p<0.0001)$ compared with euglycemic rats.

increased placental weight $(\mathrm{p}<0.001)$ and significantly decreased fetal weight $(\mathrm{p}<0.0001$; figure 1D). Fetal to placental weight ratio, a measure of placental efficiency, was significantly decreased in hyperglycemic rats compared with the euglycemic control $(\mathrm{p}<0.0001$; figure 1D). No significant sex differences were observed in placental weight, fetal weight, or the fetal to placental weight ratio (online supplementary figure 2) on gd 18.5. Taken together, these results suggest that exposure to STZ-induced hyperglycemia, 
a hallmark of poorly controlled diabetes, led to placental deficits and, as a result, diminished fetal size.

\section{Expanded junctional zone contributes to increased placental size}

To further identify the specific placental malformations resulting from diabetes exposure, placentation sites were harvested and their histological organization were assessed using immunofluorescence staining with a pancytokeratin antibody. The pan-cytokeratin antibody preferentially detects trophoblast cells within the junctional zone of the placenta (figure 1E). In contrast, trophoblastassociated cytokeratin expression in the labyrinth zone is diminished relative to the junctional zone because of its substantial mesenchymal cell content. Using this immunohistochemical approach, junctional and labyrinth zone areas were quantified. Area quantification confirmed that both junctional and labyrinth zones are significantly expanded in placentas from hyperglycemic pregnancies compared with euglycemic pregnancies $(\mathrm{p}<0.001, \mathrm{p}<0.0001$; figure $1 \mathrm{~F})$. Expanded regions of low cell density, corresponding to a greater number of glycogen cells, fill the junctional zones of placentation sites from hyperglycemic rats and contribute to the observed increase in junctional zone area.

\section{Diabetes alters junctional zone gene expression profiles}

To gain insights into molecular mechanisms associated with the expanded junctional zone of placentas from dams with poorly controlled diabetes, we performed RNAseq to assess transcriptomic profiles from euglycemic and hyperglycemic pregnant rats on gd 13.5 ( $n=4$ /group). By gd 13.5, placental zonation is established, but infiltration of invasive trophoblast cells into the uterine parenchyma has yet to occur. Analysis of the RNA-seq data identified 89 significantly upregulated transcripts and 319 significantly downregulated transcripts in the hyperglycemic group compared with the euglycemic group (figure 2A). Pathway analysis encompassing all significantly altered transcripts identified several cell processes implicated in junctional zone changes in response to uncontrolled diabetes. Inflammation, cell death, and glucose metabolism disorder were highly associated with the upregulated transcripts (figure 2A). Conversely, significantly downregulated transcripts in the hyperglycemia group included inflammatory response, cellular growth and proliferation, macronutrient metabolism, cell signaling, as well as cell survival and differentiation. Several significantly upregulated and downregulated genes were validated by reverse transcription quantitative polymerase chain reaction (RT-qPCR) (figure 2B). Among the differentially expressed transcripts were two prolactin family
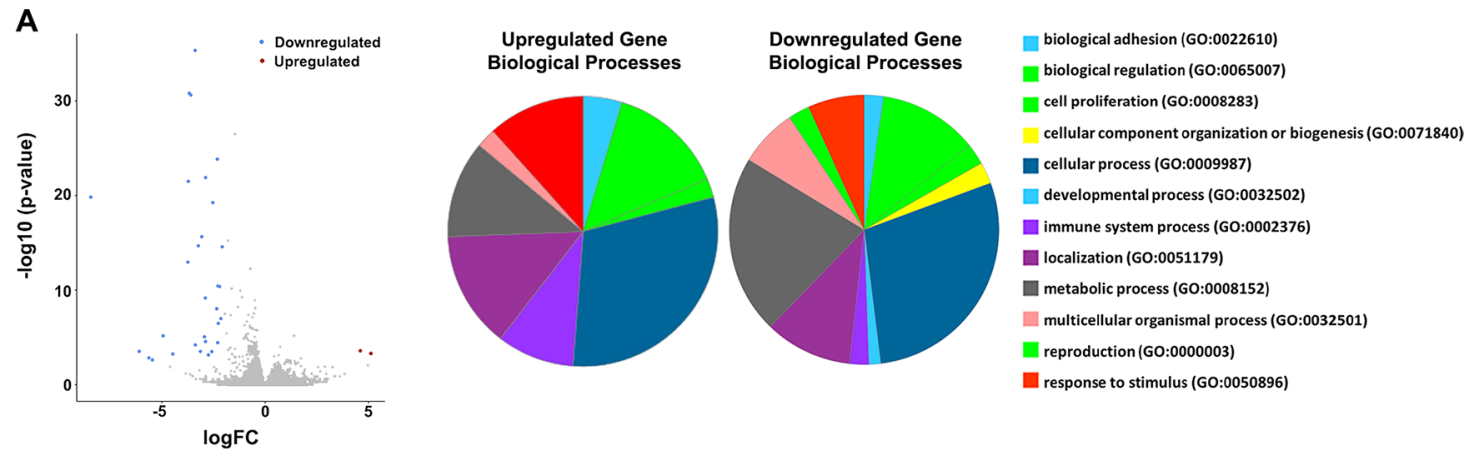

B
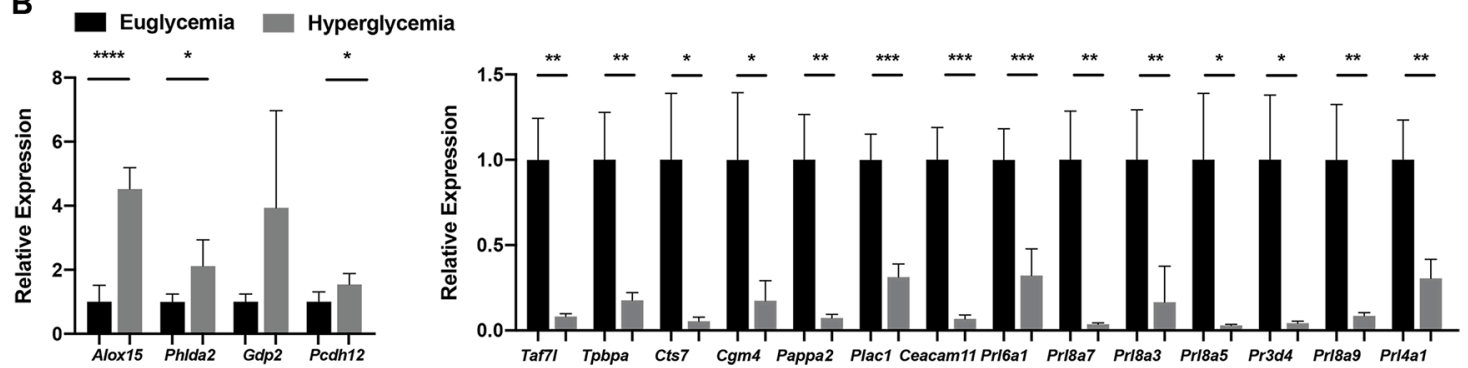

Figure 2 Diabetes exposure alters junctional zone gene expression. (A) Volcano plot depicting differential gene expression identified by RNA sequencing analysis of junctional zones from euglycemic and hyperglycemic pregnant dams ( $\mathrm{n}=4$ per group). Each dot represents one gene. The x-axis depicts logarithm to base 2 of the fold change (logFC). Significantly downregulated (blue) and upregulated (red) genes are highlighted. Pie charts display the percentage of upregulated (top) or downregulated (bottom) genes, identified by the RNA sequencing analysis, that contribute to the top gene ontology (GO) terms (GO: \#) identified by Biological Process analysis. (B) Levels of select transcripts were quantified by reverse transcription quantitative polymerase chain reaction (RT-qPCR) to validate results obtained from RNA sequencing analysis. RT-qPCR validation was performed on a number of transcripts (x-axes), which were significantly upregulated (left) or downregulated (right) in junctional zones from animals with hyperglycemia (gray) compared with euglycemia (black; $n=6$ per group, ${ }^{*} p<0.05$, ${ }^{\star *} p<0.01$, ${ }^{\star \star *} p<0.001,{ }^{* \star \star *} p<0.0001$ ). 
members, Prl3d4 and Prl8a5, which are known markers of junctional zone development. ${ }^{16}$ PRL3D4 protein levels were quantified in junctional zone tissue collected from euglycemic and hyperglycemic pregnancies on $\mathrm{gd}$ 13.5 and gd 18.5 by western blotting. PRL3D4 was significantly decreased in junctional zone tissues from hyperglycemic pregnancies at both gd 13.5 and 18.5 (gd 13.5, $\mathrm{p}<0.0001$, gd 18.5, $\mathrm{p}<0.01$; online supplementary figure 3). Diminished PRL3D4 expression was also observed within junctional zone compartments of placentation site cross sections from hyperglycemic pregnancies (online supplementary figure 3). Overall, the findings indicate that poorly controlled diabetes induces dysregulation of junctional zone development.

\section{Elevated glucose disrupts rat TS cell differentiation}

One of the primary manifestations of diabetes, and a phenomenon we observe in our rat model, is hyperglycemia. To determine if elevated glucose impacts TS cell behavior, we cultured rat TS cells in $5 \mathrm{mM}$ glucose (approximating a euglycemic state) or $25 \mathrm{mM}$ glucose (approximating a hyperglycemic state) and assessed the ability of TS cells to undergo cell differentiation, focusing on junctional zone transcripts affected by hyperglycemia in vivo (figure 2B). Transcript levels were used to determine the extent of differentiation throughout a 16-day differentiation time course. In $5 \mathrm{mM}$ glucose, rat TS cells begin to induce expression of genes indicative of trophoblast cell differentiation (Alox15, Prl3d4, Tpbpa, Taf $7 l$ ) with increasing transcript levels observed until day 16 (figure 3A). Under TS cell-differentiating conditions in the presence of elevated glucose $(25 \mathrm{mM})$, Alox 15 is induced to a significantly greater extent on days 12 and 16 of differentiation compared with culture in $5 \mathrm{mM}$ glucose exposure (figure 3A). In contrast, rat TS cells differentiated in $25 \mathrm{mM}$ glucose exhibit significantly decreased expression of other differentiation-associated transcripts at day 16 of culture, including Prl3d4, Plac1, Tpbpa, Taf71, and Prl8a5 (figure 3A). A similar inhibition of PRL8A5 and PRL3D4 protein expression was also observed in $25 \mathrm{mM}$ glucose-exposed TS cells (figure 3B,C; online supplementary figure 4). Overall, the results indicate that elevated glucose impairs TS cell differentiation and parallel in vivo placental responses to hyperglycemia.

\section{Diabetes impairs intrauterine trophoblast invasion}

The junctional zone is prominently altered in our rat model of poorly controlled diabetes (figures 1 and 2) and represents the site of progenitors for invasive trophoblast lineage development within the rat placenta. ${ }^{6} 21$ Therefore, we sought to determine whether hyperglycemia impacts development of the invasive trophoblast lineage in vivo. To assess this, we quantified the depth and extent of cytokeratin-positive trophoblast cell invasion into the uterus and measured transcript levels of invasive trophoblast-specific markers (Prl5a1, Prl7b1, Plac1) in dissected metrial glands, the site of intrauterine trophoblast cell invasion.
Quantitation of the depth of trophoblast invasion by cytokeratin staining in euglycemic and hyperglycemic pregnant rats was assessed on gd 18.5. Endovascular trophoblast invasion depth was similar in euglycemic and hyperglycemic pregnant rats (figure 4B). However, interstitial trophoblast invasion was almost completely absent on gd 18.5 in hyperglycemic rats compared with euglycemic rats (figure 4A). Total area of trophoblast invasion, including endovascular and interstitial, was decreased in hyperglycemic compared with euglycemic pregnant rats (figure 4C). Prl5a1 and Prl7b1 are specific markers of intrauterine invasive trophoblast cells, ${ }^{22} 23$ and Plac1 has recently been implicated as an intrinsic regulator of the invasive trophoblast lineage. ${ }^{24} \operatorname{Prl5a1}$, Prl7b1, and Plac1 transcript levels were significantly decreased within the uterine-placental interface of hyperglycemic versus euglycemic pregnancies (figure 4D). Collectively, the results indicate that hyperglycemia adversely affects development of the invasive trophoblast lineage.

\section{Hyperglycemia and placenta plasticity}

A fundamental property of the placenta is its plasticity. ${ }^{25}$ The placenta possesses the ability to adapt to a wide range of environmental challenges that could threaten pregnancy. ${ }^{625}$ Adjustments in trophoblast-uterine spiral artery interactions are a key component of placental adaptations to environmental stressors. ${ }^{46}$ Hypoxia has proven to be an effective tool to elicit adaptations in the development of the maternal-fetal interface. ${ }^{17}$ Since hyperglycemia affected both junctional zone and invasive trophoblast cell lineage development, we sought to determine the impact of hyperglycemia on hypoxia-induced placental plasticity.

We used an experimental paradigm of hypoxia exposure in the pregnant rat proven to activate endovascular trophoblast invasion and trophoblast-guided uterine spiral artery remodeling. ${ }^{17}$ Euglycemic and hyperglycemic rats were exposed to ambient conditions $(\sim 21 \%$ oxygen) or hypoxia ( $10.5 \%$ oxygen) beginning on $\mathrm{gd}$ 6.5 and sacrificed on gd 13.5 (figure 5A). The extent of STZ-induced hyperglycemia was similar in ambient and hypoxia exposed pregnant rats (figure 5B). Hypoxia exposure significantly decreased uterine + conceptus weight in hyperglycemic compared with euglycemic pregnant rats at gd 9.5 ( $p<0.01$; figure 5A) but did not adversely affect conceptus viability. Conceptuses from euglycemic pregnant rats exposed to ambient or hypoxic conditions and conceptuses from hyperglycemic pregnant rats exposed to ambient conditions survived to gd 9.5 (figure 5D). However, by gd 13.5 all hyperglycemic rats exposed to hypoxia showed complete pregnancy loss $(\mathrm{p}<0.0001$; figure 5E). These findings prompted examination of conceptus development in each group at an earlier stage of gestation (gd 9.5). Histological analysis of gd 9.5 conceptuses demonstrated that development of the primordial placenta, referred to as the ectoplacental cone, was adversely affected in hyperglycemic pregnancies exposed to hypoxia (figure $5 \mathrm{~F}$ ), which was verified 
A
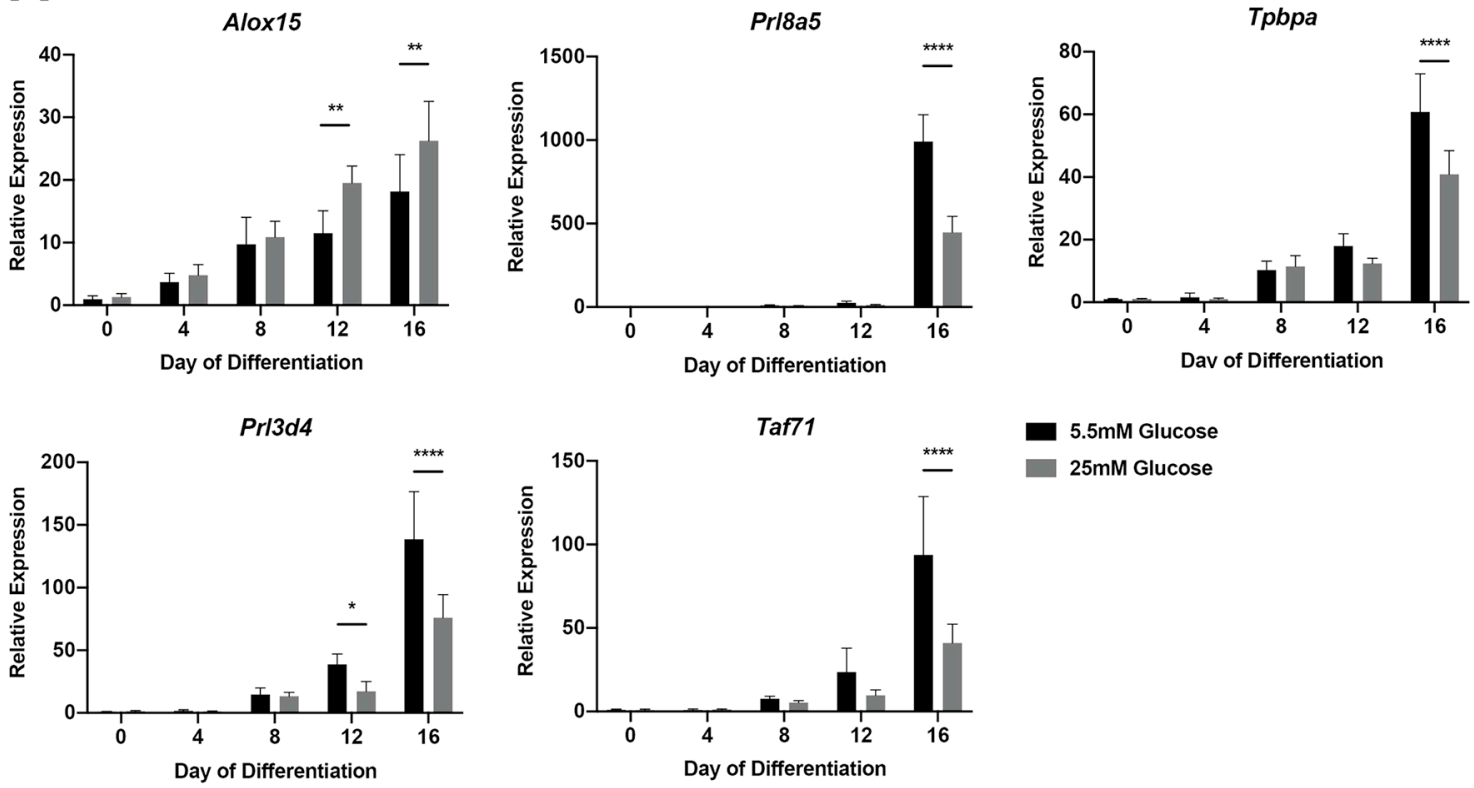

B
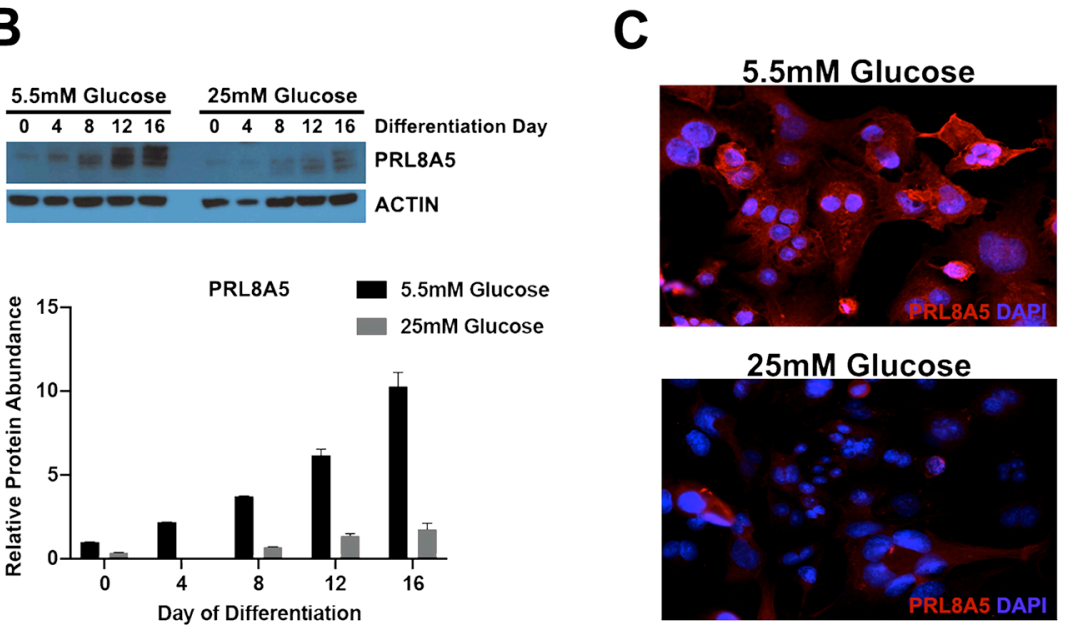

Figure 3 High glucose modulates rat trophoblast stem cell (TS) differentiation. (A) Rat TS cells were cultured in differentiation medium containing $5 \mathrm{mM}$ glucose (black) or $25 \mathrm{mM}$ glucose (gray) for 16 days. RNA was isolated from both treatment groups at five time points (days $0,4,8,12$, and 16). Significant differences are denoted by asterisks ( $n=6$ per group; ${ }^{*} p<0.05,{ }^{* *} p<0.01$, $\left.{ }^{* * * *} \mathrm{p}<0.0001\right)$. Western blot depicting PRL8A5 (B) protein levels in rat TS cells cultured in differentiation medium containing $5 \mathrm{mM}$ glucose (black) or $25 \mathrm{mM}$ glucose (gray). Protein was collected from both treatment groups at five time points (days 0 , 4, 8, 12, and 16). PRL8A5 protein levels were quantified with ImageJ (National Institutes of Health), normalized to beta actin (ACTIN), and graphed as "Relative Protein Abundance" ( $y$-axis; $n=5$ per group). (C) Immunocytochemical analysis of PRL8A5 (red) expression in rat TS cells cultured in differentiation medium containing $5 \mathrm{mM}$ glucose or $25 \mathrm{mM}$ glucose for 16 days. 4',6-diamidino-2-phenylindole (DAPI, blue) marks cell nuclei.

by the quantification of ectoplacental cone surface area $(\mathrm{p}<0.0001$; figure $5 \mathrm{G})$ and diminished proliferation, as measured by phosphohistone $\mathrm{H} 3$ immunoreactivity $(\mathrm{p}<0.0001$; figure $5 \mathrm{H})$. Thus, hyperglycemia adversely affects placental adaptations to hypoxia.

\section{High glucose blunts TS cell responses to low oxygen}

To determine whether the interaction of hyperglycemia and hypoxia was directly altering trophoblast lineage development, we investigated the impact of glucose on TS cell responses to low oxygen in vitro. Transcript levels of known TS cell hypoxia-responsive genes, including IL33 and CD200, were measured by RT-qPCR. ${ }^{27}$ IL 33 and
CD200 transcript concentrations were similar in TS cells cultured in $5 \mathrm{mM}$ or $25 \mathrm{mM}$ glucose exposed to ambient conditions $(\mathrm{p}<0.01$; online supplementary figure 5$)$. In contrast, exposure to low oxygen significantly induced expression of $I L 33$ and $C D 200$ in $5 \mathrm{mM}$ glucose $(\mathrm{p}<0.01)$ but $I L 33$ and $C D 200$ were not induced by hypoxia in $25 \mathrm{mM}$ glucose $(\mathrm{p}<0.01)$. We also examined the interactive effects of glucose and hypoxia on TS cell number. TS cell viability was not altered by the culture conditions (figure 5I); however, low oxygen stimulated TS cell proliferation in $5 \mathrm{mM}$ glucose, but not in $25 \mathrm{mM}$ glucose $(p<0.0001$; figure 5I). These in vitro results support the 
A

\section{Euglycemia}

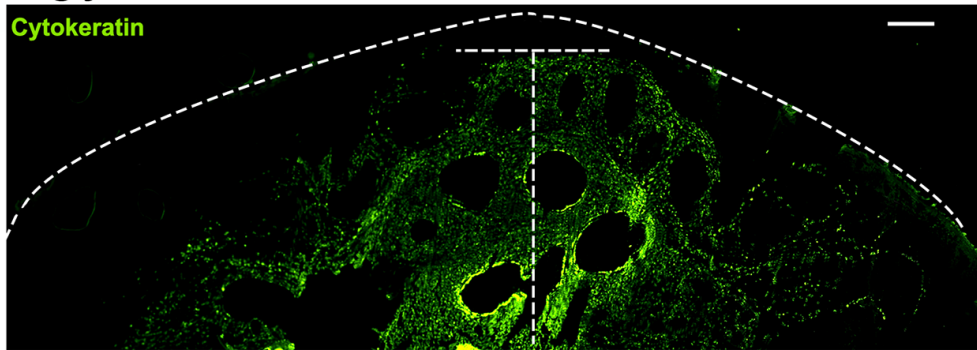

Hyperglycemia

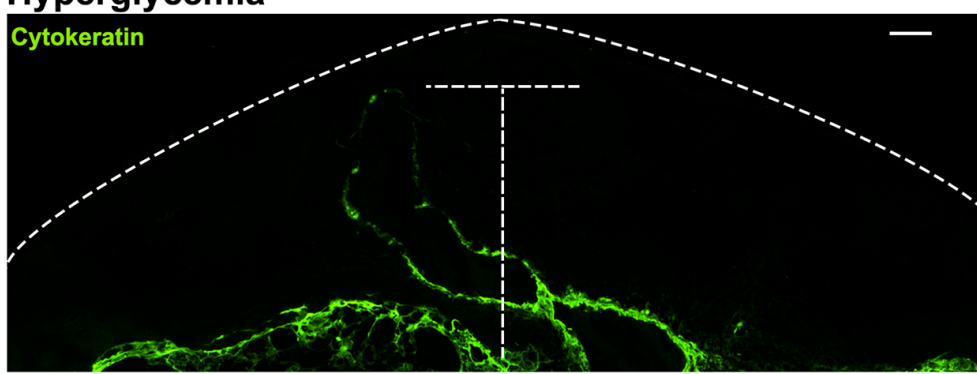

D

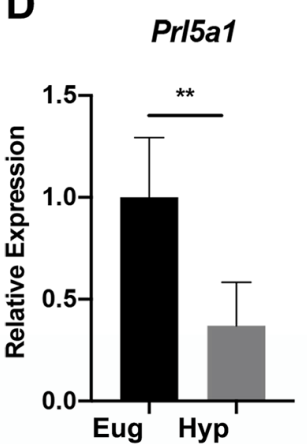

Prl7b1

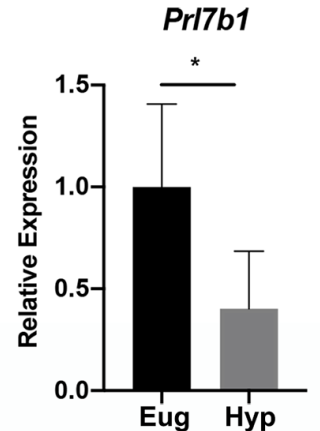

B

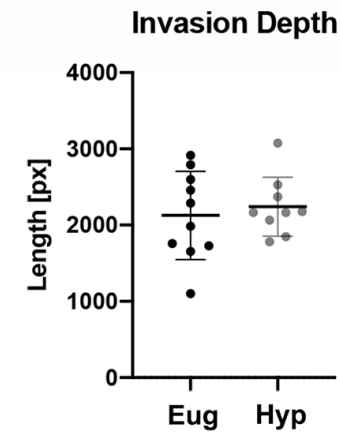

C

Invasion Area

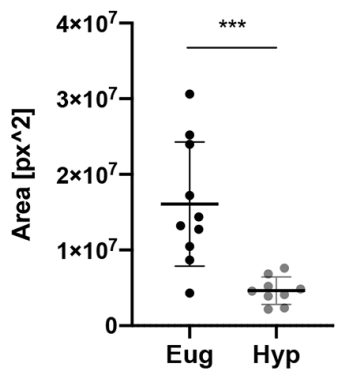

Figure 4 Diabetes impairs uterine trophoblast invasion and spiral artery remodeling. (A) Placentation sites collected from euglycemic (Eug) and hyperglycemic (Hyp) rats on gestation day (gd) 18.5 were sectioned and stained with a cytokeratinspecific antibody (green) for immunohistochemical analysis and visualization of trophoblast cells and placental compartments. Brightly labeled, cytokeratin-positive trophoblast cells invade interstitially and endovascularly into the metrial gland compartment. Images were acquired with a 10x objective. The scale bars represent $250 \mu \mathrm{m}$. (B) Depth of intrauterine trophoblast cell invasion was quantified in ImageJ (National Institutes of Health $(\mathrm{NIH})$ as an invasion index, or the distance of distal-most endovascular cytokeratin-positive cell relative to the trophoblast giant cell layer of the chorioallantoic placenta. Invasion depth was similar between Eug and Hyp pregnant rats ( $n=10$ Eug, $n=9$ Hyp; $p>0.05)$. (C) Total area of trophoblast invasion, including endovascular and interstitial, was quantified in ImageJ $(\mathrm{NIH})$. Hyp significantly reduced interstitial, but not endovascular, trophoblast invasion compared with Eug ( $n=10$ Eug, $n=9 H y p ; p<0.001)$. (D) RNA was isolated from uterineplacental interface tissue dissected from Eug and Hyp pregnant dams on gd 18.5. Prl5a1, Prl7b1, and Plac1 transcripts were significantly downregulated in the uterine-placental interface collected from Hyp animals (gray) compared with Eug animals (black; $n=6$ per group, ${ }^{\star} \mathrm{p}<0.05,{ }^{* *} \mathrm{p}<0.01$ ).

in vivo observations that diabetes (hyperglycemia) alters placental cell plasticity through, at least in part, direct actions on trophoblast cell development.

\section{DISCUSSION}

Animal models have been effectively used to investigate diabetes and pregnancy. ${ }^{28}$ In this study, we examined the impact of poorly controlled diabetes on placental and fetal development in the rat. We provide evidence that placental development in a hyperglycemic state is not optimal. Hyperglycemia leads to the formation of an abnormally large, inefficient placenta that adversely affects fetal growth and that does not adapt well to an adverse maternal environment. Our results suggest that some of the effects of hyperglycemia on placentation are due to the direct actions of high glucose on trophoblast cell behavior.

In this report, we have not attempted to mimic a specific human syndrome or disease state with the rat. Fundamental differences between rat and human pregnancy render such a task highly problematic. Placentationrelated events that transpire during rodent gestation best equate to physiological changes that occur during the first trimester of human pregnancy. ${ }^{29}$ From a superficial 
A

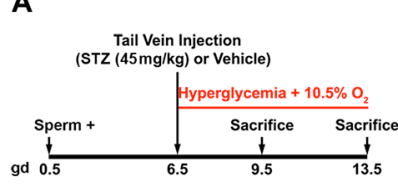

C

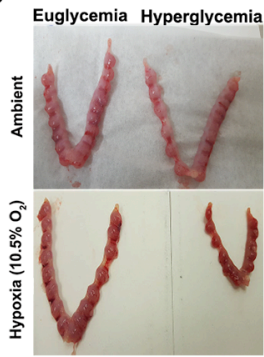

D

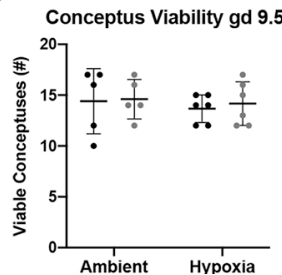

B Non-fasting Blood Glucose gd 13.5
F

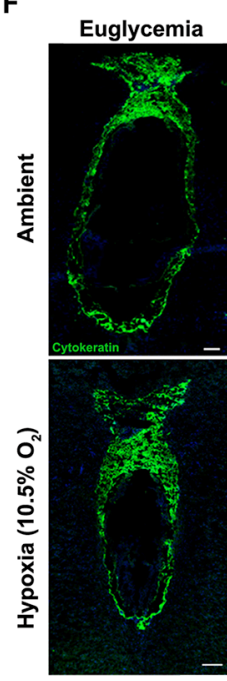

G

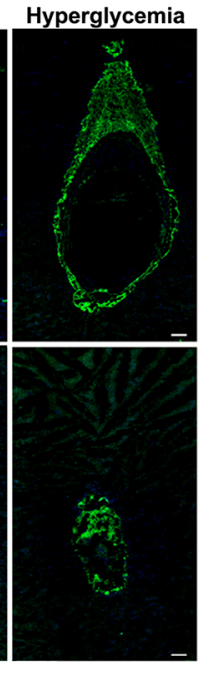

H
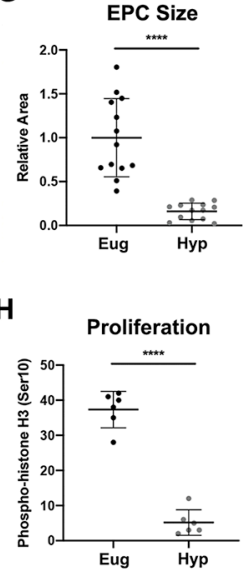

E

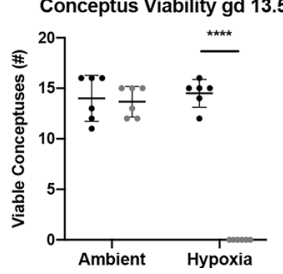

Figure 5 Hyperglycemia diminishes conceptus viability and ectoplacental cone development (EPC) in pregnant rats exposed to hypoxia. (A) Schematic depicting the experimental design for streptozotocin (STZ) treatment and hypoxia exposure of pregnant rats. Half of the rats were maintained in ambient air housing and the other half were housed in a gas-regulated chamber to mimic hypoxia (10.5\% (vol/vol) oxygen) until sacrifice on gestation day (gd) 9.5 or 13.5 for tissue harvest. Photomicrographs of whole uteri collected from euglycemia (Eug) and hyperglycemia (Hyp) animals housed in ambient air or hypoxia. (B) Glucose was measured on gd 13.5 in rats housed in ambient air and hypoxia. Hyp (gray) possessed significantly increased blood glucose levels compared with Eug (black) animals in both ambient air and hypoxia ( $\mathrm{n}=6$ for all groups except $\mathrm{n}=8$ for Hyp in hypoxia, $\mathrm{p}<0.0001$ ). (C) Uterine weight was significantly reduced in Hyp animals compared with Eug animals when exposed to hypoxia $n=5$ for ambient and $n=6$ for hypoxia, $p<0.01$ ). (D) The average number of viable conceptuses was not different between Eug (black) and Hyp (gray) animals housed in ambient air or hypoxia on gd 9.5 ( $n=5$ for ambient and $n=6$ for hypoxia, $p>0.05$ ). (E) Viable conceptuses were not recovered in Hyp animals housed in hypoxia on gd 13.5, resulting in a significant difference between treatment groups ( $n=6$ per group, $p<0.0001$ ). (F) Placentation sites were collected on gd 9.5, sectioned, and stained for cytokeratin (green) and 4',6-diamidino-2-phenylindole (DAPI, blue) from Eug and Hyp rats housed in ambient air or hypoxia $\left(10.5 \% \mathrm{O}_{2}\right)$. (G) EPC size, quantified as relative area of cytokeratin-positive cells, is significantly decreased in Hyp animals (gray) compared with Eug animals (black) $(n=13$ per group, $p<0.0001)$. (H) Hyp significantly decreased EPC cell proliferation, which was measured as the number of phospho-histone H3 positive cells ( $\mathrm{n}=6$ per group, $\mathrm{p}<0.0001)$. (I) Rat trophoblast stem cells were cultured in medium containing $5 \mathrm{mM}$ glucose (black) or $25 \mathrm{mM}$ glucose (gray) and were maintained in either ambient air or exposed to low oxygen $\left(1.5 \% \mathrm{O}_{2}\right)$ for 24 hours and the number of viable cells quantified. Significant differences are denoted by asterisks $(n=6$ per group, $p<0.0001)$.

perspective, placentation appears to be different in the rat and human; however, this view is biased by the use of distinct, species-specific nomenclature. In fact, rat and human placentas are highly conserved. ${ }^{212530}$ Both species possess hemochorial placentas that perform the same critical tasks. Central to those tasks are specialized trophoblast cells that transform the uterine environment, including restructuring the uterine spiral arteries, to regulate delivery of maternal nutrients to the developing fetus. ${ }^{25}$ Although the hyperglycemia we observe with our model of STZ-induced beta cell damage may best reflect poorly controlled type 1 diabetes in pregnancy, the goal of the experimentation outlined in this report was to gain broader insights into the impact of glucose dysregulation on the morphogenesis of the placenta.

Disruptions in placental structure are a common feature of hyperglycemia. ${ }^{28} 31$ Our observations of placentomegaly are consistent with earlier reports examining the effects of diabetes mellitus on placental development in the mouse and rat. ${ }^{32-35}$ An expansion of glycogen cells within the junctional zone compartment has been a common finding. ${ }^{36}$ Diabetes in humans is associated with alterations in placental surface area, volume, intervillous space, terminal villi, syncytiotrophoblast, fibrinoid areas, and glycogen deposits. ${ }^{31}$

A key element of human placentation is the entry of trophoblast cells into the uterus where they restructure the uterine parenchyma, including remodeling of uterine spiral arteries. ${ }^{4525}$ This form of deep placentation is also observed in the rat. ${ }^{21} 22$ Two invasive trophoblast cell populations are evident. Interstitial invasive trophoblast cells are situated between the uterine vasculature, whereas endovascular invasive trophoblast cells replace the endothelium of the uterine spiral arterioles. ${ }^{425}$ In 
our experimentation, we observed that hyperglycemia preferentially led to disruptions in the interstitial invasive trophoblast population. Impairments in intrauterine trophoblast cell invasion have also been observed in the biobreeding diabetes-prone rat model. ${ }^{38}$ There is also some indication that trophoblast cell invasion is adversely affected in humans with diabetes. ${ }^{39} 40$ Specific mechanisms underlying invasive trophoblast cell reorganization of the uterine parenchyma is not well appreciated. Furthermore, we know little of how hyperglycemia affects differentiation of the invasive trophoblast cell lineage.

Some insights into the effects of hyperglycemia on placentation may be gleaned from the transcript profile of junctional zone placental tissue from euglycemic and hyperglycemic rat pregnancies. In addition to the dysregulation of species-specific members of the prolactin family (Prl3d4, Prl8a5, Prl8a9), transcripts from the pregnancy-specific glycoprotein family $(\operatorname{Cgm} 4)$, and the cathepsin family $(\mathrm{Cts} 7)$, were also identified as dysregulated in response to hyperglycemia. Downregulated transcripts, such as Taf7l, Pappa2, and Plac1, could contribute to hyperglycemia-induced placental dysmorphogenesis. Taf $7 l$ encodes a member of a family of proteins that associate with the TATA-binding protein (TBP) and is essential for TBP binding to the TATA element and transcription. ${ }^{41}$ TAF7L has been implicated in sperm development and the regulation of adipocyte differentiation, ${ }^{42}{ }^{43}$ but not placentation. Pappa 2 encodes a protease targeting insulin-like growth factor (IGF)-binding proteins, ${ }^{44}$ which modulate the activity of IGFs,${ }^{45}$ known regulators of placental growth. ${ }^{46}$ However, disruption of Pappa 2 in the mouse has only subtle effects on male reproduction and modest effects on postnatal growth with no reported adverse effects on placental development. ${ }^{47}{ }^{48}$ In contrast, placenta specific 1 (Plac1), a highly conserved X-linked gene, possesses pronounced effects on placentation. ${ }^{49} 50$ Targeted disruption of Plac1 in the mouse leads to placentomegaly, including an expanded junctional zone. ${ }^{40}$ Downregulation of Plac1 expression in the placenta associated with hyperglycemia fits well with the phenotype of the Plac1 null mouse. Thus, PLAC1 represents a candidate mediator of the effects of hyperglycemia on placentation.

A healthy placenta exhibits plasticity and can adapt to a range of environmental challenges. ${ }^{625}$ Hypoxia is an environmental challenge that can elicit placental adaptations, including the stimulation of trophoblast invasion and spiral artery remodeling. ${ }^{17}$ Hypoxia is also a physiological regulator of placentation ${ }^{26}$ and has been identified as a key regulator of invasive/extravillous trophoblast cell differentiation. ${ }^{182751}$ Dual exposure to hyperglycemia and hypoxia in the rat was not compatible with pregnancy. Pregnancies were terminated due to growth arrest at the ectoplacental cone stage of placentation. Cellular responses to low oxygen were blunted when TS cells were cultured in high glucose implicating direct interactive effects of oxygen tension and glucose on trophoblast cells. High altitude is associated with low oxygen tensions and increased risks for pregnancy complications. ${ }^{52}$ The combination of high altitude and diabetes may be particularly problematic to the development of an effective hemochorial placenta.

In summary, our data generated using an in vivo STZ model of diabetes and in vitro analyses of TS cells support the hypothesis that exposure to hyperglycemia during gestation induces placental alterations that result in impaired fetal outcomes. The initiation of hyperglycemia post-embryo implantation was a key element of our experimental design and distinct from other animal models of diabetes. ${ }^{28}$ Our findings strengthen earlier observations that intrauterine exposure to hyperglycemia alters structural formation and glycogen cell accumulation within the placenta. New insights into the impairment of trophoblast differentiation and invasion in placentation sites from dams with hyperglycemia highlights a potential mechanism by which fetal growth restriction may occur in this model. We also found that placentas from hyperglycemic pregnancies lacked the requisite plasticity to adapt to the stress of hypoxia exposure and led to pregnancy loss. These findings reinforce the damaging effects of poorly controlled diabetes on placental formation and pregnancy outcomes as well as the experimental value of the rat model in the investigation of hemochorial placentation and critical events regulating trophoblast invasion and spiral artery remodeling.

\section{Author affiliations}

${ }^{1}$ Department of Pathology and Laboratory Medicine, University of Kansas Medical Center, Kansas City, Kansas, USA

${ }^{2}$ Institute for Reproduction and Perinatal Research, University of Kansas Medical Center, Kansas City, Kansas, USA

${ }^{3}$ Center for Perinatal Research, Children's Mercy Research Institute, Children's Mercy, Kansas City, Missouri, USA

${ }^{4}$ Department of Pediatrics, University of Kansas Medical Center, Kansas City, Kansas, USA

${ }^{5}$ Department of Obstetrics and Gynecology, University of Kansas Medical Center, Kansas City, Kansas, USA

Acknowledgements We thank Stacy 0xley, Priscilla Nechrebecki, and Brandi Miller for their assistance. We also thank staff of the University of Kansas Medical Center Laboratory Animal Resources.

Contributors JN, KMV, MJS conceived and designed the research; JN, KMV, RLS, MES, and KI performed experiments and analyzed data; JN, KMV, KI, and MJS interpreted results of experiments; KMV and KI prepared figures; KMV drafted manuscript; KMV and MJS edited and revised manuscript; JN, KMV, KI and MJS approved the final version of manuscript.

Funding The research was supported by a postdoctoral fellowship to JN from the American Diabetes Association (1-16-PMF-012), an NRSA postdoctoral fellowship to KMV from the NIH (F32HD096809), grants from the NIH (HD020676, HD079363, HD099638), and the Sosland Foundation.

Competing interests MJS is the guarantor of this work and, as such, had full access to all the data in the study and takes responsibility for the integrity of the data and the accuracy of the data analysis.

Patient consent for publication Not required.

Ethics approval The study obtained ethics approval by the KUMC Animal Care and Use Committee (Approval No 2019-2495). All rats were maintained in accordance with institutional policies for the care and use of vertebrate animals in research using protocols approved by the KUMC Animal Care and Use Committee.

Provenance and peer review Not commissioned; externally peer reviewed. 
Data availability statement Data are available in a public, open access repository. Data are available on reasonable request. The datasets generated and analyzed during the current study are available in the Gene Expression Omnibus website (https://www.ncbi.nlm.nih.gov/geo/ ; accession no. GSE144276). All data generated and analyzed during this study are included in the published article and the online supplementary files. Resources generated and analyzed during the current study are available from the corresponding authors on reasonable request.

Open access This is an open access article distributed in accordance with the Creative Commons Attribution Non Commercial (CC BY-NC 4.0) license, which permits others to distribute, remix, adapt, build upon this work non-commercially, and license their derivative works on different terms, provided the original work is properly cited, appropriate credit is given, any changes made indicated, and the use is non-commercial. See: http://creativecommons.org/licenses/by-nc/4.0/.

ORCID iD

Kaela M Varberg http://orcid.org/0000-0001-9672-4158

\section{REFERENCES}

1 Getahun D, Nath C, Ananth CV, et al. Gestational diabetes in the United States: temporal trends 1989 through 2004. Am J Obstet Gynecol 2008;198:525.e1-5.

2 Hay WW. Placental-fetal glucose exchange and fetal glucose metabolism. Trans Am Clin Climatol Assoc 2006;117:321-40.

3 Burton GJ, Fowden AL, Thornburg KL. Placental origins of chronic disease. Physiol Rev 2016;96:1509-65.

4 Pijnenborg R, Vercruysse L, Hanssens M. The uterine spiral arteries in human pregnancy: facts and controversies. Placenta 2006;27:939-58.

5 Brosens I, Pijnenborg R, Vercruysse L, et al. The "Great Obstetrical Syndromes" are associated with disorders of deep placentation. Am J Obstet Gynecol 2011;204:193-201.

6 Soares MJ, Chakraborty D, Kubota K, et al. Adaptive mechanisms controlling uterine spiral artery remodeling during the establishment of pregnancy. Int J Dev Biol 2014;58:247-59.

7 Chang C-W, Wakeland AK, Parast MM, et al. Trophoblast lineage specification, differentiation and their regulation by oxygen tension. $J$ Endocrinol 2018;236:R43-56.

8 Wu KK, Huan Y. Streptozotocin-Induced diabetic models in mice and rats. Curr Protoc Pharmacol 2008;Chapter 5:5.47.1-5.47.14.

9 Like A, Rossini A. Streptozotocin-induced pancreatic insulitis: new model of diabetes mellitus. Science 1976;193:415-7.

10 Weide LG, Lacy PE. Low-dose streptozocin-induced autoimmune diabetes in islet transplantation model. Diabetes 1991;40:1157-62.

11 Van Assche FA, Aerts L, Holemans K. Metabolic alterations in adulthood after intrauterine development in mothers with mild diabetes. Diabetes 1991;40(Suppl 2):106-8.

12 López-Soldado I, Herrera E. Different diabetogenic response to moderate doses of streptozotocin in pregnant rats, and its long-term consequences in the offspring. Exp Diabesity Res 2003;4:107-18.

13 Nteeba J, Kubota K, Wang W, et al. Pancreatic prolactin receptor signaling regulates maternal glucose homeostasis. J Endocrinol 2019;241:71-83.

14 Ain R, Konno T, Canham LN, et al. Phenotypic analysis of the rat placenta. Methods Mol Med 2006;121:295-313.

15 Asanoma K, Rumi MAK, Kent LN, et al. FGF4-dependent stem cells derived from rat blastocysts differentiate along the trophoblast lineage. Dev Biol 2011;351:110-9.

16 Deb S, Roby KF, Faria TN, et al. Identification and immunochemical characterization of a major placental secretory protein related to the prolactin-growth hormone family, prolactin-like protein-C. Endocrinology 1991;128:3066-72.

17 Rosario GX, Konno T, Soares MJ. Maternal hypoxia activates endovascular trophoblast cell invasion. Dev Biol 2008;314:362-75.

18 Chakraborty D, Rumi MAK, Konno T, et al. Natural killer cells direct hemochorial placentation by regulating hypoxia-inducible factor dependent trophoblast lineage decisions. Proc Natl Acad Sci U S A 2011:108:16295-300.

19 Dhakal P, Rumi MAK, Kubota K, et al. Neonatal progesterone programs adult uterine responses to progesterone and susceptibility to uterine dysfunction. Endocrinology 2015;156:3791-803.

20 Deb S, Faria TN, Roby KF, et al. Identification and characterization of a new member of the prolactin family, placental lactogen-I variant. J Biol Chem 1991;266:1605-10.

21 Soares MJ, Chakraborty D, Karim Rumi MA, et al. Rat placentation: an experimental model for investigating the hemochorial maternalfetal interface. Placenta 2012;33:233-43.
22 Ain R, Canham LN, Soares MJ. Gestation stage-dependent intrauterine trophoblast cell invasion in the rat and mouse: novel endocrine phenotype and regulation. Dev Biol 2003;260:176-90.

23 Wiemers DO, Ain R, Ohboshi S, et al. Migratory trophoblast cells express a newly identified member of the prolactin gene family. $J$ Endocrinol 2003;179:335-46.

24 Chang W-L, Yang Q, Zhang H, et al. Role of placenta-specific protein 1 in trophoblast invasion and migration. Reproduction 2014;148:343-52.

25 Soares MJ, Varberg KM, lqbal K, et al. Hemochorial placentation: development, function, and adaptations. Biol Reprod 2018;99:196-211.

26 Burton GJ. Oxygen, the Janus gas; its effects on human placental development and function. J Anat 2009;215:27-35.

27 Chakraborty D, Cui W, Rosario GX, et al. HIF-KDM3AMMP12 regulatory circuit ensures trophoblast plasticity and placental adaptations to hypoxia. Proc Natl Acad Sci U S A 2016;113:E7212-21.

28 Jawerbaum A, White V. Animal models in diabetes and pregnancy. Endocr Rev 2010;31:680-701.

29 Soncin F, Khater M, To C, et al. Comparative analysis of mouse and human placentae across gestation reveals species-specific regulators of placental development. Development 2018;145. doi:10.1242/dev.156273. [Epub ahead of print: 29 Jan 2018].

30 Pijnenborg R, Robertson WB, Brosens I, et al. Review article: trophoblast invasion and the establishment of haemochorial placentation in man and laboratory animals. Placenta 1981;2:71-91.

31 Carrasco-Wong I, Moller A, Giachini FR, et al. Placental structure in gestational diabetes mellitus. Biochim Biophys Acta BBA - Mol Basis Dis 2020;1866:165535.

32 Salbaum JM, Kruger C, Zhang X, et al. Altered gene expression and spongiotrophoblast differentiation in placenta from a mouse model of diabetes in pregnancy. Diabetologia 2011;54:1909-20.

33 Zorn TMT, Zúñiga M, Madrid E, et al. Maternal diabetes affects cell proliferation in developing rat placenta. Histol Histopathol 2011;26:1049-56.

34 Yu Y, Singh U, Shi W, et al. Influence of murine maternal diabetes on placental morphology, gene expression, and function. Arch Physiol Biochem 2008;114:99-110.

35 Farias PS, dos S Souza K, Fioretto ET, et al. Maternal diabetes affects rat placental morphology and pregnancy. Endocrine 2014;45:497-501.

36 Gewolb IH, Merdian W, Warshaw JB, et al. Fine structura abnormalities of the placenta in diabetic rats. Diabetes 1986;35:1254-61.

37 Ne'eman Z, Barash V, Rosenmann E, et al. Localization of glycogen in the placenta of diabetic rats: a light and electron microscopic study. Placenta 1987;8:201-8.

38 Groen B, Uuldriks GA, de Vos P, et al. Impaired trophoblast invasion and increased numbers of immune cells at day 18 of pregnancy in the mesometrial triangle of type 1 diabetic rats. Placenta 2015;36:142-9.

39 Cawyer CR, Horvat D, Leonard D, et al. Hyperglycemia impairs cytotrophoblast function via stress signaling. Am J Obstet Gynecol 2014;211:541.e1-8.

40 Heidari Z, Mahmoudzadeh-Sagheb H, Narouei M, et al. Effects of gestational diabetes mellitus on stereological parameters and extravillous trophoblast cells of placenta compared to the control group. J Obstet Gynaecol 2019;39:928-33.

41 Goodrich JA, Tjian R. TBP-TAF complexes: selectivity factors for eukaryotic transcription. Curr Opin Cell Biol 1994;6:403-9.

42 Cheng Y, Buffone MG, Kouadio M, et al. Abnormal sperm in mice lacking the Taf7l gene. Mol Cell Biol 2007;27:2582-9.

43 Zhou H, Kaplan T, Li Y, et al. Dual functions of TAF7L in adipocyte differentiation. Elife 2013;2:e00170.

44 Andrew M, Liao L, Fujimoto M, et al. PAPPA2 as a Therapeutic Modulator of IGF-I Bioavailability: in Vivo and in Vitro Evidence. $J$ Endocr Soc 2018:2:646-56.

45 Bach LA. IGF-binding proteins. J Mol Endocrinol 2018;61:T11-28.

46 Sferruzzi-Perri AN, Sandovici I, Constancia M, et al. Placental phenotype and the insulin-like growth factors: resource allocation to fetal growth. J Physiol 2017;595:5057-93.

47 Conover CA, Boldt HB, Bale LK, et al. Pregnancy-associated plasma protein-A2 (PAPP-A2): tissue expression and biological consequences of gene knockout in mice. Endocrinology 2011:152:2837-44.

48 Christians JK, King AY, Rogowska MD, et al. Pappa2 deletion in mice affects male but not female fertility. Reprod Biol Endocrinol 2015;13:109. 
49 Jackman SM, Kong X, Fant ME. Plac1 (placenta-specific 1) is essential for normal placental and embryonic development. Mol Reprod Dev 2012;79:564-72.

50 Muto M, Fujihara Y, Tobita T, et al. Lentiviral vector-mediated complementation restored fetal viability but not placental hyperplasia in Plac1-deficient mice. Biol Reprod 2016;94:6.
51 Wakeland AK, Soncin F, Moretto-Zita M, et al. Hypoxia directs human extravillous trophoblast differentiation in a hypoxia-inducible factor-dependent manner. Am J Pathol 2017:187:767-80.

52 Zamudio S. The placenta at high altitude. High Alt Med Biol 2003;4:171-91. 